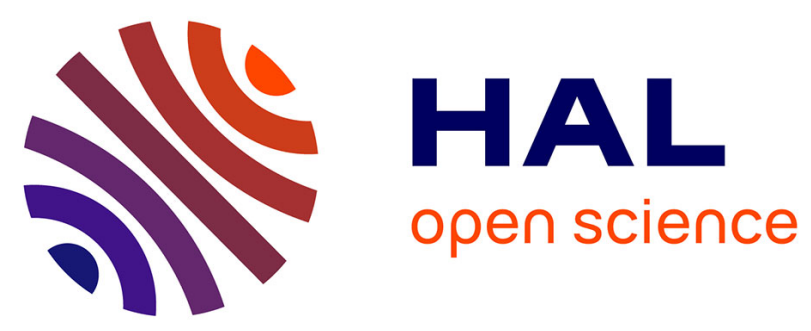

\title{
Synthesis and characterization of diruthenaborane analogues of pentaborane(11) and hexaborane(10)
}

B. Joseph, S. Gomosta, S.K. Barik, S.K. Sinha, Thierry Roisnel, V. Dorcet, J.-F. Halet, S. Ghosh

\section{> To cite this version:}

B. Joseph, S. Gomosta, S.K. Barik, S.K. Sinha, Thierry Roisnel, et al.. Synthesis and characterization of diruthenaborane analogues of pentaborane(11) and hexaborane(10). Journal of Organometallic Chemistry, 2018, 865, pp.29-36. 10.1016/j.jorganchem.2017.12.011 . hal-01809146

HAL Id: hal-01809146

https://hal-univ-rennes1.archives-ouvertes.fr/hal-01809146

Submitted on 14 Jun 2018

HAL is a multi-disciplinary open access archive for the deposit and dissemination of scientific research documents, whether they are published or not. The documents may come from teaching and research institutions in France or abroad, or from public or private research centers.
L'archive ouverte pluridisciplinaire HAL, est destinée au dépôt et à la diffusion de documents scientifiques de niveau recherche, publiés ou non, émanant des établissements d'enseignement et de recherche français ou étrangers, des laboratoires publics ou privés. 


\title{
Synthesis and characterization of diruthenaborane analogues of pentaborane(11) and hexaborane $(10)^{\Phi}$
}

\author{
Benson Joseph ${ }^{a}$, Suman Gomosta ${ }^{a}$, Subrat Kumar Barik ${ }^{a}$, Soumya Kumar Sinha ${ }^{a}$, Thierry Roisnel $^{b}$, \\ Vincent Dorcet ${ }^{b}$, Jean-François Halet ${ }^{b} *$,SundargopalGhosh ${ }^{a} *$
}

${ }^{a}$ Department of Chemistry, Indian Institute of Technology Madras, Chennai 600036, India
${ }^{\mathrm{b}}$ Institut des Sciences Chimiques de Rennes, UMR 6226, CNRS-Ecole Nationale Supérieure de
Chimie de Rennes-Université de Rennes 1, F-35042 Rennes Cedex, France
$\Phi_{\text {Dedicated to our good friend and colleague Prof. Narayan Hosmane on the occasion of his } 70^{\text {th }}}$

Birthday.

Keywords: cluster compound, borane, metallaborane, phenyl-chalcogenoborate, ruthenaheteroborane

\begin{abstract}
:
In an attempt to synthesize expanded-cage metallaheteroboranes containing heavier chalcogen atoms, the reaction of diruthenaborane analogue of pentaborane $(9)$, nido-[1,2- $\left.(\mathrm{Cp} * \mathrm{RuH})_{2} \mathrm{~B}_{3} \mathrm{H}_{7}\right](\mathbf{1})$ with phenyl-chalcogenoborates $\mathrm{Li}\left[\mathrm{BH}_{3}(\mathrm{EPh})\right](\mathrm{E}=\mathrm{S}$, Se or Te) was carried out. Thermolysis of nido-1 with $\mathrm{Li}\left[\mathrm{BH}_{3}(\mathrm{SPh})\right]$ led to the formation of the dimetalla-pentaborane(11) analogue arachno$\left[(\mathrm{Cp} * \mathrm{Ru})_{2} \mathrm{~B}_{3} \mathrm{H}_{8}(\mathrm{SPh})\right]$ (2). In parallel to the formation of $\mathbf{2}$, the reaction also yielded three B-H functionalized compounds, namely $\left[\left(\mathrm{Cp}^{*} \mathrm{Ru}\right)_{2} \mathrm{~B}_{4} \mathrm{H}_{7}(\mathrm{Ph})\right] \quad(3), \quad\left[(\mathrm{Cp} * \mathrm{Ru})_{2} \mathrm{~B}_{4} \mathrm{H}_{7}(\mathrm{Cl})\right] \quad$ (4) and $\left[(\mathrm{Cp} * \mathrm{Ru})_{2} \mathrm{~B}_{4} \mathrm{H}_{6}(\mathrm{SPh})(\mathrm{Cl})\right](\mathbf{5})$. On the other hand, reaction of $\mathbf{1}$ with $\mathrm{Li}\left[\mathrm{BH}_{3}(\mathrm{SePh})\right]$ led to the formation of the diruthenium analogue of hexaborane(10) nido-[( $\left.\mathrm{Cp} * \mathrm{Ru})_{2} \mathrm{~B}_{4} \mathrm{H}_{9}(\mathrm{SePh})\right](6)$, whereas $\mathrm{Li}\left[\mathrm{BH}_{3}(\mathrm{TePh})\right]$ yielded the capped nido-pentagonal-pyramidal $\left[(\mathrm{Cp} * \mathrm{Ru})_{2} \mathrm{~B}_{4} \mathrm{H}_{6} \mathrm{Te}\right]$ (7). Compound 7 is a rare ruthenaborane cluster containing a heavier chalcogen element (Te). All the compounds were characterized by mass spectrometry and ${ }^{1} \mathrm{H},{ }^{1} \mathrm{H}\left\{{ }^{11} \mathrm{~B}\right\},{ }^{11} \mathrm{~B}\left\{{ }^{1} \mathrm{H}\right\}$ and ${ }^{13} \mathrm{C}\left\{{ }^{1} \mathrm{H}\right\} \mathrm{NMR}$ spectroscopy. The
\end{abstract}


solid state X-ray structures of all the compounds were unequivocally established by crystallographic analysis. Additionally, the electronic properties of compound 2 were analyzed.

\section{Introduction}

The chemistry of heteroboranes mostly embraces carboranes and metallocarboranes [1] and to a lesser extent thiaboranes and thiametallaboranes [2]. By contrast, heteroborane clusters that contain heavier group-16 elements as part of the cluster constituents are far less numerous [3]. However, because of the availability of few synthetic methods that are of potential for the synthesis of heteroborane and metallaheteroborane compounds [4,5], several compounds have been synthesized and structurally characterized. The chemistry of this sub-area of transition metal complexes of boron have received significant attention both from the structure/bonding and reactivity viewpoints. As a result, we and others have synthesized many interesting polyhedral cage compounds that include clusters beyond icosahedral cage [6-9]. As a part of our interest in synthesizing metallaheteroboranes containing heavier chalcogen atoms, we have recently synthesized a series of group 5, 6 and 9 dimetallaheteroborane clusters, namely, nido-[(CpNb $\left.)_{2} \mathrm{BHSe}_{4}\right]$, nido- $\left[(\mathrm{Cp} * \mathrm{M})_{2} \mathrm{~B}_{4} \mathrm{H}_{4} \mathrm{E}_{2}\right](\mathrm{M}=\mathrm{Mo}$ and $\mathrm{W}$ and $\mathrm{E}=\mathrm{S}, \mathrm{Se}$ and $\mathrm{Te})$, and nido- $\left[(\mathrm{Cp} * \mathrm{Co})_{2} \mathrm{~B}_{2} \mathrm{H}_{2} \mathrm{E}_{2}\right](\mathrm{E}=\mathrm{S}$ and $\mathrm{Se})$ [10-12], by the reactions of metal polychlorides with monoborane reagents in presence of dichalcogenide ligands or chalcogen powders. As structurally characterized examples of diruthenaheteroboranes are rare, we became interested in investigating the use of different chalcogen sources for the generation of new types of cluster systems. Upon the availability of one of the very common and reactive metallaborane nido-[1,2-(Cp* RuH $\left.)_{2} \mathrm{~B}_{3} \mathrm{H}_{7}\right]$ (nido-1), which is very prone to cluster expansion reaction, [13] we thought to utilize this as a precursor for the synthesis of new ruthenaheteroboranes. Herein, we report some new metallaheteroboranes containing heavier chalcogen atoms obtained from mild pyrolysis of the diruthenaborane analogue of pentaborane $(9)$, nido-[1,2-(Cp* $\left.\mathrm{RuH})_{2} \mathrm{~B}_{3} \mathrm{H}_{7}\right]$, with phenyl-chalcogenoborates $\mathrm{Li}\left[\mathrm{BH}_{3} . \mathrm{EPh}\right](\mathrm{E}=\mathrm{S}, \mathrm{Se}$ or $\mathrm{Te})$. 


\section{Results and discussion}

\subsection{Reaction of nido-1 with $\mathrm{Li}_{[}\left[\mathrm{BH}_{3}(\mathrm{SPh})\right]$}

As shown in Scheme 1, compounds 2-5 were obtained in moderate yields from the thermolysis of nido-1 with excess of $\mathrm{Li}\left[\mathrm{BH}_{3}(\mathrm{SPh})\right]$. Although these compounds were produced in a mixture, chromatographic workup using thin-layer chromatography (TLC) plates enabled us to isolate them selectively in pure form as orange and yellow solids. Details of the spectroscopic and structural characterization of these compounds are discussed below.

(Scheme 1. near here)

\subsubsection{Arachno-[(Cp*Ru $\left.)_{2} B_{3} H_{8}(S P h)\right], 2$}

Compound 2 was isolated as a yellow solid in $20 \%$ yield and was characterized by multinuclear NMR, IR spectroscopy, mass spectrometry and single crystal X-ray diffraction studies. The mass spectrum of 2 shows a parent ion peak at $m / z 625.1$ for $[\mathrm{M}+\mathrm{H}]^{+}$. The room temperature ${ }^{11} \mathrm{~B}\left\{{ }^{1} \mathrm{H}\right\}$ NMR spectrum shows the presence of three boron environments at $\delta=30.2,4.9$ and $-1.7 \mathrm{ppm}$ in a 1:1:1 ratio. Besides the $\mathrm{BH}$ terminal hydrogen atoms, the ${ }^{1} \mathrm{H}$ NMR spectrum exhibits five different signals at $\delta=-2.89,-3.05,-10.45,-13.76$ and -17.03 ppm due to $\mathrm{B}-H-\mathrm{B}, \mathrm{Ru}-H-\mathrm{B}, \mathrm{Ru}-H-\mathrm{Ru}$ and $\mathrm{Ru}-$ $H_{t}$ protons. Furthermore, analysis of both ${ }^{1} \mathrm{H}$ and ${ }^{13} \mathrm{C}\left\{{ }^{1} \mathrm{H}\right\}$ NMR spectra suggests two equivalents of Cp* ligands. An absorption band at $799.8 \mathrm{~cm}^{-1}$ in the IR spectrum indicates the presence of the $\mathrm{Ru}-\mathrm{S}$ stretching vibration.

(Fig. 1. near here)

In order to confirm these spectroscopic assignments and to determine the solid state structure of $\mathbf{2}$, an X-ray structure analysis was undertaken. As shown in Fig. 1, its molecular structure can be viewed as a dimetalla analogue of pentaborane(11) of structural type I-II, shown in Table 1 [14], with a bridged $\mathrm{SPh}$ unit between Ru2 and B10 centers. The framework structure of 2 which consists of an eight-sep, five-vertex arachno $\left[\mathrm{Ru}_{2} \mathrm{~B}_{3}\right]$ cluster, can indeed be predicted by the polyhedral skeleton electron pair (sep) counting rules $[15,16]$. Formation of $\mathbf{2}$ from the square pyramidal nido-1 
species might occur via the co-ordination of $\mathrm{S}$ to the ruthenaborane followed by the breaking of one of the Ru-B bonds. The interatomic Ru1-Ru2 distance ( $2.975 \AA$ ) is relatively long probably because of the presence of a bridging hydrogen atom and comparable to the related compound $\mathbf{I}[14 \mathrm{~b}]$.

(Table 1. near here)

(Fig. 2. near here)

To shed some light on the electronic structure and bonding of $\mathbf{2}$, density functional theory (DFT) calculations were carried out on model 2' (Cp analogue of 2) at the PBE0/Def2TZVP level of theory (see computational details in the Experimental section). The optimized structural parameters (Table S1, see supporting information (SI)) and calculated NMR chemical-shifts $\left({ }^{1} \mathrm{H}\right.$ and $\left.{ }^{11} \mathrm{~B}\right)$ (Table S2, see SI) of 2' compare well to those experimentally observed for $\mathbf{2}$. For example, the computed Ru2S9 and B10-S9 distances, 2.358 and $1.925 \AA$, match well with the experimentally observed distances of 2.386(15) and 1.940(7) $\AA$, respectively. Additionally, the DFT calculations are very helpful in confirming accurately the positions of the bridging hydrogen atoms. One of the notable features of compound 2 is the presence of a five-membered metallaheterocycle (B10-B11-B12-Ru2-S9). A topological analysis [17] reveals the existence of bond critical points (BCPs) along the Ru2-S9 and B10-S9 bond paths. The Ru2-S9 bond path is characterized with positive $\rho$ and $\nabla^{2} \rho(\mathrm{r})$ values $(\rho(\mathrm{r})$ : 0.085 a.u. and $\nabla^{2} \rho$ (r): 0.220 a.u.) (Fig. 2 a, Table S3 (SI)). The presence of BCPs for B10-B11 $(\rho($ r): 0.117 a.u. and $\nabla^{2} \rho\left(\right.$ r): 0.416 a.u.), B11-B12 ( $\rho\left(\right.$ r): 0.113 a.u. and $\nabla^{2} \rho($ r): 0.470 a.u.) and Ru2-B12 ( $\rho\left(\right.$ r): 0.125 a.u. and $\nabla^{2} \rho($ r): -0.194 a.u.) bond paths (Fig. 2b, Table S3, (SI)) are also observed.

The Wiberg bond index (WBI) values calculated on the basis of a natural bond orbital (NBO) analysis predict significant bonding interactions between the $\mathrm{Ru}-\mathrm{S}, \mathrm{B}-\mathrm{S}$ and $\mathrm{B}-\mathrm{B}$ atoms $\left(\mathrm{WBI}_{\mathrm{Ru2}-\mathrm{S} 9}=\right.$ $0.74, \mathrm{WBI}_{\mathrm{B} 10-\mathrm{S} 9}=0.93, \mathrm{WBI}_{\mathrm{B} 10-\mathrm{B} 11}=0.51, \mathrm{WBI}_{\mathrm{B} 11-\mathrm{B} 12}=0.53$ and $\left.\mathrm{WBI}_{\mathrm{B} 12-\mathrm{Ru} 2}=0.44\right)$, indicating complete involvement of all the five atoms of the five-membered $\mathrm{RuSB}_{3}$ metallaheterocycle. Additionally, a molecular orbital analysis reveals the existence of a substantial $\sigma$-bonding interaction between $\mathrm{Ru} 1$ and $\mathrm{Ru} 2$ illustrated by the occupied $\mathrm{d}_{\mathrm{z}}{ }^{2}$ orbital-character HOMO-6 (Fig. 2c) and the 
corresponding vacant antibonding LUMO (Fig. 2e) of 2', both heavily localized on the two metal centers.

\subsection{2. $\left[\left(C p^{*} R u\right)_{2} B_{4} H_{7}(P h)\right], 3,\left[\left(C p^{*} R u\right)_{2} B_{4} H_{7}(C l)\right], 4$, and $\left[(C p * R u)_{2} B_{4} H_{6}(S P h)(C l)\right], 5$}

In parallel to the formation of compound 2, treatment of nido-1 with $\mathrm{Li}_{[}\left[\mathrm{BH}_{3}(\mathrm{SPh})\right]$ also yielded compounds 3-5 (Scheme 1) in low yields. The ${ }^{11} \mathrm{~B}\left\{{ }^{1} \mathrm{H}\right\}$ NMR spectra of them show the presence of different resonances. $(\mathbf{3}: \delta=124.8,33.9,21.6$ and -31.6 ppm; $\mathbf{4}: \delta=128.8,15.7$ and -10.9 ppm; 5: $\delta$ $=133.8,23.04,19.9$ and $-7.9 \mathrm{ppm})$. The analysis of both ${ }^{1} \mathrm{H}$ and ${ }^{13} \mathrm{C}\left\{{ }^{1} \mathrm{H}\right\}$ NMR spectra of 3-5 confirms the presence of $\mathrm{Cp}^{*}$ ligands. In addition, ${ }^{1} \mathrm{H}$ NMR spectra of 3-5 reveals distinct up-field peaks due to the presence of $\mathrm{B}-H-\mathrm{B}, \mathrm{Ru}-H-\mathrm{B}$, and $\mathrm{Ru}-H$-Ru protons (Table 2 ).

The single-crystal X-ray structures of compounds 3-5, shown in Fig. 3, confirm the structural inferences based on spectroscopic results. Further, the existence of similar spectral pattern $\left({ }^{1} \mathrm{H}\right.$ and ${ }^{11} \mathrm{~B}\left\{{ }^{1} \mathrm{H}\right\}$ NMR) of compounds 3-5 led us to compare them with the existing ruthenaborane cluster $\left[(\mathrm{Cp} * \mathrm{Ru})_{2} \mathrm{~B}_{4} \mathrm{H}_{7}(\mu-\mathrm{H})\right][13]$ and its trimetalla derivative $\left[(\mathrm{Cp} * \mathrm{Ru})_{3} \mathrm{~B}_{3} \mathrm{H}_{7}(\mu-\mathrm{H})\right]$ [18] (Table 2). They all possess a capped nido square-pyramidal cluster core with the identical sep count of 7. The Ru1$\mathrm{Ru} 2$ bond lengths in $\mathbf{3 ,} \mathbf{4}$ and $\mathbf{5}$ are slightly longer than those observed in $\left[(\mathrm{Cp} * \mathrm{Ru})_{2} \mathrm{~B}_{4} \mathrm{H}_{7}(\mu-\mathrm{H})\right][13]$ and $\left[(\mathrm{Cp} * \mathrm{Ru})_{3} \mathrm{~B}_{3} \mathrm{H}_{7}(\mu-\mathrm{H})\right][18,19]$.

(Fig. 3. near here)

(Table 2. near here)

\subsection{Reaction of nido-1 with $\mathrm{Li}\left[\mathrm{BH}_{3}(\mathrm{SePh})\right]$}

In light of the synthesis of $\mathbf{2 - 5}$, under similar reaction conditions the reaction of nido-1 with $\mathrm{Li}\left[\mathrm{BH}_{3}(\mathrm{SePh})\right]$ yielded nido-[(Cp* $\left.\left.\mathrm{Ru}\right)_{2} \mathrm{~B}_{4} \mathrm{H}_{9}(\mathrm{SePh})\right], \mathbf{6}$, along with 3 (Scheme 1). The mass spectrum of 6 shows the parent ion peak at $m / z, 707.0981$ for $[\mathrm{M}+\mathrm{Na}]^{+}$. The ${ }^{11} \mathrm{~B}\left\{{ }^{1} \mathrm{H}\right\}$ NMR spectrum of 6 exhibits three resonance peaks at $\delta=20.3,19.5$ and $4.9 \mathrm{ppm}$. The ${ }^{1} \mathrm{H}$ and ${ }^{13} \mathrm{C}\left\{{ }^{1} \mathrm{H}\right\}$ NMR spectra show the presence of $\mathrm{Cp}$ * protons. Further, the ${ }^{11} \mathrm{H}\left\{{ }^{11} \mathrm{~B}\right\}$ NMR spectra reveals the presence of three 
chemical shifts at $\delta=4.60,3.02$ and 2.51 ppm confirming the presence of three $\mathrm{B}-H$ terminal protons.

As shown in Fig. 4, the solid state X-ray structure of compound $\mathbf{6}$ can be considered as a diruthenium analogue of hexaborane(10) in which the apical $\mathrm{BH}$ and one of the basal $\mathrm{BH}$ vertices have been replaced by $\{\mathrm{Cp} * \mathrm{Ru}\}$ and $\mathrm{SePh}$ fragments. The core $\mathrm{Ru}_{2} \mathrm{~B}_{4}$ geometry of 6 is similar to that of the nido-[(Cp* Ru $\left.)_{2} \mathrm{~B}_{4} \mathrm{H}_{10}\right][13 \mathrm{a}]$ and nido- $\left[\left(\mathrm{Cp}^{*} \mathrm{Ir}\right)_{2} \mathrm{~B}_{4} \mathrm{H}_{7}(\mu-\mathrm{H})\right]$ [19] species. They all possess the same electron count of eight sep (Table 3). Again, the formation of $\mathbf{6}$ from the square pyramidal compound nido-1 can be explained in terms of cluster expansion reaction followed by replacement of a $\mathrm{BH}$ group by a $\mathrm{B}(\mathrm{SePh})$ moiety. Both the $\mathrm{Ru}-\mathrm{Ru}$ and $\mathrm{B}-\mathrm{Se}$ bonds are marginally longer as compared to other selenaborane clusters [20].

(Fig. 4. near here)

(Table 3. near here)

\subsection{Reaction of nido-1 with $\mathrm{Li}_{[}\left[\mathrm{BH}_{3}(\mathrm{TePh})\right]$}

The compound $\left[(\mathrm{Cp} * \mathrm{Ru})_{2} \mathrm{~B}_{4} \mathrm{H}_{7} \mathrm{Te}\right], \mathbf{7}$, was isolated as a purple solid from the reaction of nido-1 with $\mathrm{Li}\left[\mathrm{BH}_{3}(\mathrm{TePh})\right]$ along with 3 (Scheme 1). It was characterized by multinuclear NMR spectroscopy and single-crystal X-ray diffraction studies. The molecular ion peak for 7 was observed at $m / z=655.0420$ for $[\mathrm{M}+\mathrm{H}]^{+}$. The ${ }^{11} \mathrm{~B}\left\{{ }^{1} \mathrm{H}\right\}$ NMR spectrum shows the presence of four different chemical shifts at $\delta=80.3,23.1,17.5$, and $-17.5 \mathrm{ppm}$. Besides the $\mathrm{B}-\mathrm{H}_{\mathrm{t}}$ and the two types of $\mathrm{Cp}^{*}$ protons, the ${ }^{1} \mathrm{H}$ NMR also shows the presence of B- $H$-B up-fielded protons at $\delta=-4.24$ and -12.51 ppm respectively in $1: 1$ ratio. The ${ }^{13} \mathrm{C}\left\{{ }^{1} \mathrm{H}\right\}$ NMR spectrum that shows two different chemical shifts for the $\mathrm{Cp}^{*}$ ligands confirm their different chemical environment.

(Fig. 5. near here)

The structural assignment, based on the X-ray crystallographic data, confirms the geometry of 7 (Fig. 5). Though six-vertex clusters with capped-square pyramidal geometry [18] and corresponding seven-vertex clusters with capped octahedral geometry $[13,21]$ are known, 7 is the first example with 
a capped-pentagonal pyramidal geometry with a heavy chalcogen (Te) atom occupying a vertex (Fig.

5). The Te atom is inserted into the $\left[(\mathrm{Cp} * \mathrm{Ru})_{2}(\mu-\mathrm{H})_{2} \mathrm{~B}_{3} \mathrm{H}_{7}\right]$ cluster cage forming a pentagonal pyramidal core followed by a BH moiety capping the pentagonal pyramid cluster. With a sep count of eight, the boron-capped nido-pentagonal pyramidal cluster 7 is isoelectronic with $\mathrm{B}_{6} \mathrm{H}_{10}$. The Ru1-Te1 and Ru2-Te1 distance of 2.532(3) and 2.584(3) $\AA$ in 7 can be compared to the Ru-Te distance $(2.549(2) \AA)$ observed in a ruthenatelluraborane cluster closo-[2- $\left(\eta^{6}-\mathrm{C}_{6} \mathrm{Me}_{6}\right)-1,2-$ $\left.\mathrm{TeRuB}_{10} \mathrm{H}_{10}\right][3 \mathrm{a}]$.

\section{Conclusion}

Results discussed here have demonstrated that many transient metallaborane intermediates can be stabilized by phenyl-chalcogenoborates $\mathrm{Li}\left[\mathrm{BH}_{3}(\mathrm{EPh})\right](\mathrm{E}=\mathrm{S}$, Se or Te). With $\mathrm{E}=\mathrm{S}$, Se, the arachno- $\left[(\mathrm{Cp} * \mathrm{Ru})_{2} \mathrm{~B}_{3} \mathrm{H}_{8}(\mathrm{SPh})\right]$ and nido- $\left[\left(\mathrm{Cp}^{*} \mathrm{Ru}\right)_{2} \mathrm{~B}_{4} \mathrm{H}_{9}(\mathrm{SePh})\right]$ dimetallaboranes, analogues of penta-borane(11) and hexaborane(10), respectively, were synthesized and structurally characterized. With $\mathrm{E}=\mathrm{Te}$, the isolation and structural characterization of $\left[(\mathrm{Cp} * \mathrm{Ru})_{2} \mathrm{~B}_{4} \mathrm{H}_{6} \mathrm{Te}\right]$ provided a rare example of metallatelluraborane. Additionally, with $\mathrm{E}=\mathrm{S}$, the clusters $\left[(\mathrm{Cp} * \mathrm{Ru})_{2} \mathrm{~B}_{4} \mathrm{H}_{7}(\mathrm{Ph})\right]$, $\left[(\mathrm{Cp} * \mathrm{Ru})_{2} \mathrm{~B}_{4} \mathrm{H}_{7}(\mathrm{Cl})\right]$ and $\left[(\mathrm{Cp} * \mathrm{Ru})_{2} \mathrm{~B}_{4} \mathrm{H}_{6}(\mathrm{SPh})(\mathrm{Cl})\right]$ were characterized, allowing a direct structural comparison with their parent molecule nido-[(Cp*RuH $\left.)_{2} \mathrm{~B}_{3} \mathrm{H}_{7}\right]$, without perturbations caused by additional main group fragments in the cluster cage and represent B-H functionalized products.

\section{Experimental details}

\subsection{General procedures and instrumentation}

All the reactions were performed under an Ar atmosphere using Schlenk line technique. The solvents were distilled under $\mathrm{Ar}$ atmosphere before use. $\left[\mathrm{Cp} * \mathrm{RuCl}_{2}\right]_{2}[22], 1$ [13a], $\mathrm{Li}\left[\mathrm{BH}_{3}(\mathrm{EPh})\right](\mathrm{E}$ $=\mathrm{S}, \mathrm{Se}$ or Te) [23], were prepared according to the literature methods. $\mathrm{Cp} * \mathrm{H},\left[\mathrm{LiBH}_{4} \cdot \mathrm{THF}\right],\left[\mathrm{Ph}_{2} \mathrm{~S}_{2}\right]$, $\left[\mathrm{Ph}_{2} \mathrm{Se}_{2}\right]$ and $\left[\mathrm{Ph}_{2} \mathrm{Te}_{2}\right]$ were obtained commercially and used as received. $\left[\mathrm{Bu}_{4} \mathrm{~N}\left(\mathrm{~B}_{3} \mathrm{H}_{8}\right)\right]$ as external 
reference for ${ }^{11} \mathrm{~B}$ NMR was synthesized according to the reported procedure [24]. Thin-layer chromatographies were performed on alumina-supported silica gel TLC plates $(250 \mathrm{~mm}$ diameter, Merck TLC plates). NMR spectra were recorded on Bruker $500 \mathrm{MHz}$ spectrometers. The residual solvent protons were used as reference $\left(\delta, \mathrm{ppm}, \mathrm{d}_{6}\right.$-benzene, $\left.7.16, \mathrm{CDCl}_{3}, 7.26\right)$, while a sealed tube containing $\left[\mathrm{Bu}_{4} \mathrm{~N}\left(\mathrm{~B}_{3} \mathrm{H}_{8}\right)\right]$ in $\mathrm{d}_{6}$-benzene $\left(\delta_{\mathrm{B}}, \mathrm{ppm},-30.07\right)$ was used as an external reference for the

${ }^{11}$ B NMR. The IR spectra were recorded on a Nicolet iS10 spectrophotometer. Electrospray mass (ESI-MS) spectra were recorded using a Qtof Micro YA263 HRMS instrument.

\subsection{Synthesis of compounds $\mathbf{2 - 5}$}

In a flame-dried Schlenk tube, nido-1 $(0.13 \mathrm{~g}, 0.25 \mathrm{mmol})$ was suspended in toluene $(5 \mathrm{~mL})$ and the mixture was cooled to $-78^{\circ} \mathrm{C}$. $\mathrm{Li}\left[\mathrm{BH}_{3}(\mathrm{SPh})\right](0.130 \mathrm{~g}, 1.0 \mathrm{mmol})$ in toluene $(12 \mathrm{~mL})$ was added via cannula and then the solution was allowed to warm slowly to room temperature. The resulting reaction mixture was then heated to $80^{\circ} \mathrm{C}$ for 18 hours. The solvent was dried and the residue was extracted into n-hexane and passed through Celite. After removal of the solvent, the residue was subjected to chromatographic work-up by using TLC plates (n-hexane/ $\mathrm{CH}_{2} \mathrm{Cl}_{2}, 85: 15 \mathrm{v} / \mathrm{v}$ ) yielded yellow $\left[(\mathrm{Cp} * \mathrm{Ru})_{2} \mathrm{~B}_{3} \mathrm{H}_{8}(\mathrm{SPh})\right], \mathbf{2}(0.032 \mathrm{~g}, 20.3 \%)$, orange $\left[(\mathrm{Cp} * \mathrm{Ru})_{2} \mathrm{~B}_{4} \mathrm{H}_{7}(\mathrm{Ph})\right], \mathbf{3}(0.021 \mathrm{~g}, 13.8 \%)$ yellow $\left[(\mathrm{Cp} * \mathrm{Ru})_{2} \mathrm{~B}_{4} \mathrm{H}_{7}(\mathrm{Cl})\right], 4(0.006 \mathrm{~g}, 4.2 \%)$ and yellow $\left[(\mathrm{Cp} * \mathrm{Ru})_{2} \mathrm{~B}_{4} \mathrm{H}_{6}(\mathrm{SPh})(\mathrm{Cl})\right], 5(0.008 \mathrm{~g}$, 4.7\%). Note that the yield of compounds $\mathbf{4}$ and $\mathbf{5}$ are very less which could be resulted from the workup and separation in $n$-hexane and $\mathrm{CH}_{2} \mathrm{Cl}_{2}$ mixture.

2: MS $\left(\mathrm{ESI}^{+}\right): m / z$ calcd for $\left[\mathrm{C}_{26} \mathrm{H}_{43} \mathrm{~B}_{3} \mathrm{SRu}_{2}+\mathrm{H}\right]^{+}: 625.1$ found $625.1 ;{ }^{11} \mathrm{~B}\left\{{ }^{1} \mathrm{H}\right\}$ NMR $(160 \mathrm{MHz}$. $\left.\mathrm{CDCl}_{3}, 22{ }^{\circ} \mathrm{C}\right): \delta=30.2$ (s, 1B), 4.9 (s, 1B), $-1.7 \mathrm{ppm}(\mathrm{s}, 1 \mathrm{~B}) ;{ }^{1} \mathrm{H}$ NMR $\left(500 \mathrm{MHz}, \mathrm{CDCl}_{3}, 22{ }^{\circ} \mathrm{C}\right): \delta$ $=7.19-7.09(5 \mathrm{H}, \mathrm{Ph}), 5.79\left(\mathrm{br}, \mathrm{BH}_{t}\right), 4.28\left(\mathrm{br}, \mathrm{BH}_{t}\right), 3.16\left(\mathrm{br}, \mathrm{BH}_{t}\right), 1.85(\mathrm{~s}, 15 \mathrm{H}, \mathrm{Cp} *), 1.78(\mathrm{~s}, 15 \mathrm{H}$, Cp*),-2.96 (br, 2H, B-H-B), -10.46 (br, 1H, Ru-H-B), -13.76 (br, 1H, Ru-H-Ru), -17.03 ppm (s, 1H, $\left.\mathrm{Ru}-\mathrm{H}_{\mathrm{t}}\right) ;{ }^{1} \mathrm{H}\left\{{ }^{11} \mathrm{~B}\right\} \operatorname{NMR}\left(500 \mathrm{MHz}, \mathrm{CDCl}_{3}, 22{ }^{\circ} \mathrm{C}\right): \delta=7.17-7.04(5 \mathrm{H}, \mathrm{Ph}), 5.74\left(\mathrm{~s}, \mathrm{BH}_{t}\right), 4.26(\mathrm{~s}$, $\left.\mathrm{BH}_{t}\right), 2.99\left(\mathrm{~s}, \mathrm{BH}_{t}\right), 1.84\left(\mathrm{~s}, 15 \mathrm{H}, \mathrm{Cp}^{*}\right), 1.78\left(\mathrm{~s}, 15 \mathrm{H}, \mathrm{Cp}^{*}\right),-2.89$ (s, 1H, B-H-B), -3.05 (s, 1H, B-HB), -10.44 (s, 1H, Ru-H-B), -13.76 (s, 1H, Ru-H-Ru), -17.03 ppm (s, 1H, Ru- $\left.\mathrm{H}_{\mathrm{t}}\right) ;{ }^{13} \mathrm{C}\left\{{ }^{1} \mathrm{H}\right\}$ NMR $(125$ 
$\left.\mathrm{MHz}, \mathrm{CDCl}_{3}, 22{ }^{\circ} \mathrm{C}\right): \delta=131.6-128.3(\mathrm{~s}, \mathrm{Ph}), 96.6\left(\mathrm{~s}, C_{5} \mathrm{Me}_{5}\right), 84.6\left(\mathrm{~s}, C_{5} \mathrm{Me}_{5}\right), 12.2\left(\mathrm{~s}, \mathrm{C}_{5} \mathrm{Me}_{5}\right), 11.6$ ppm (s, $\mathrm{C}_{5} M e_{5}$ ); IR (DCM, cm $\left.{ }^{-1}\right): 799.8(\mathrm{Ru}-\mathrm{S}), 2847.3(\mathrm{C}-\mathrm{H}), 2915.8$ (Cp*), 2961.8 (Cp*).

3: HR-MS (ESI $\left.{ }^{+}\right): m / z$ calculated for $\left[\mathrm{C}_{26} \mathrm{H}_{42} \mathrm{Ru}_{2} \mathrm{~B}_{4}+\mathrm{Na}\right]^{+}: 625.1643$ found $625.1665 ;{ }^{11} \mathrm{~B}\left\{{ }^{1} \mathrm{H}\right\} \mathrm{NMR}$ $\left(160 \mathrm{MHz}, \mathrm{CDCl}_{3}, 22{ }^{\circ} \mathrm{C}\right): \delta=124.7$ (s, 1B), 33.8 (s, 1B), 21.5 (s, 1B), -31.6 ppm (s, 1B); ${ }^{1} \mathrm{H}$ NMR $\left(500 \mathrm{MHz}, \mathrm{CDCl}_{3}, 22^{\circ} \mathrm{C}\right): \delta=9.83\left(\mathrm{br}, \mathrm{BH}_{t}\right), 7.43-7.05(5 \mathrm{H}, \mathrm{Ph}), 4.74\left(\mathrm{br}, \mathrm{BH}_{t}\right), 1.87\left(\mathrm{~s}, 15 \mathrm{H}, \mathrm{Cp}^{*}\right)$, $1.83(\mathrm{~s}, 15 \mathrm{H}, \mathrm{Cp} *), 0.36\left(\mathrm{br}, 1 \mathrm{H}, \mathrm{B}-\mathrm{H}_{\mathrm{t}}\right),-2.96\left(\mathrm{pcq} \mathrm{B}^{*} \mathrm{H}_{\mathrm{t}}\right),-11.25$ (br, 1H, Ru-H-B), -12.34 (br, 1H, Ru-H-B), -15.46 ppm (s, $1 \mathrm{H}, \mathrm{Ru}-\mathrm{H}-\mathrm{Ru}) ;{ }^{1} \mathrm{H}\left\{{ }^{11} \mathrm{~B}\right\}$ NMR $\left(500 \mathrm{MHz}, \mathrm{CDCl}_{3}, 22{ }^{\circ} \mathrm{C}\right): \delta=9.64(\mathrm{br}$, $\left.\mathrm{BH}_{t}\right), 7.05-7.43(5 \mathrm{H}, \mathrm{Ph}), 4.72\left(\mathrm{~s}, \mathrm{BH}_{t}\right), 1.87(\mathrm{~s}, 15 \mathrm{H}, \mathrm{Cp} *), 1.83(\mathrm{~s}, 15 \mathrm{H}, \mathrm{Cp} *), 0.36\left(\mathrm{~s}, 1 \mathrm{H}, \mathrm{B}-\mathrm{H}_{\mathrm{t}}\right),-$ $2.94\left(\mathrm{br}, \mathrm{B}-\mathrm{H}_{\mathrm{t}}\right),-11.28(\mathrm{~s}, 1 \mathrm{H}, \mathrm{Ru}-\mathrm{H}-\mathrm{B}),-12.35$ (s, 1H, Ru-H-B), -15.46 ppm (s, 1H, Ru-H-Ru); ${ }^{13} \mathrm{C}\left\{{ }^{1} \mathrm{H}\right\} \operatorname{NMR}\left(125 \mathrm{MHz}, \mathrm{CDCl}_{3}, 22{ }^{\circ} \mathrm{C}\right): \delta=132.9-120.7(\mathrm{~s}, \mathrm{Ph}), 92.8\left(\mathrm{~s}, C_{5} \mathrm{Me}_{5}\right), 88.8\left(\mathrm{~s}, C_{5} \mathrm{Me}_{5}\right)$, 14.2 (s, $\left.\mathrm{C}_{5} M e_{5}\right), 11.3 \mathrm{ppm}\left(\mathrm{s}, \mathrm{C}_{5} M e_{5}\right)$; IR (DCM, $\left.\mathrm{cm}^{-1}\right): 2961.6,2908.6$ (Cp*), $2849.3(\mathrm{C}-\mathrm{H})$.

4: MS (ESI $\left.{ }^{+}\right): m / z$ calculated for $\left[\mathrm{C}_{20} \mathrm{H}_{37} \mathrm{Ru}_{2} \mathrm{~B}_{4} \mathrm{Cl}+\mathrm{H}\right]^{+}: 561.1$, found $561.1 ;{ }^{11} \mathrm{~B}\left\{{ }^{1} \mathrm{H}\right\}$ NMR $(160$ $\left.\mathrm{MHz}, \mathrm{CDCl}_{3}, 22{ }^{\circ} \mathrm{C}\right): \delta=128.8(\mathrm{~s}, 1 \mathrm{~B}), 15.7(\mathrm{~s}, 2 \mathrm{~B}),-10.8 \mathrm{ppm}(\mathrm{s}, 1 \mathrm{~B}) ;{ }^{1} \mathrm{H} \mathrm{NMR}\left(500 \mathrm{MHz}, \mathrm{CDCl}_{3}\right.$, $\left.22{ }^{\circ} \mathrm{C}\right): \delta=10.61\left(\mathrm{br}, \mathrm{BH}_{t}\right), 4.99\left(\mathrm{br}, 2 \mathrm{H}, \mathrm{BH}_{t}\right), 1.89\left(\mathrm{~s}, 30 \mathrm{H}, \mathrm{Cp}^{*}\right),-0.94$ (s, 1H, B-H-B), -11.31 (s, $2 \mathrm{H}, \mathrm{Ru}-\mathrm{H}-\mathrm{B}),-15.55 \mathrm{ppm}(\mathrm{s}, 1 \mathrm{H}, \mathrm{Ru}-\mathrm{H}-\mathrm{Ru}) ;{ }^{1} \mathrm{H}\left\{{ }^{11} \mathrm{~B}\right\} \mathrm{NMR}\left(500 \mathrm{MHz}, \mathrm{CDCl}_{3}, 22{ }^{\circ} \mathrm{C}\right): \delta=10.65$ (s, $\left.\mathrm{BH}_{t}\right), 4.95\left(\mathrm{~s}, 2 \mathrm{H}, \mathrm{BH}_{t}\right), 1.90$ (s, 30H, Cp*), -0.93 (s, 1H, B-H-B), -11.27 (s, 2H, Ru-H-B), -15.55 ppm (s, $1 \mathrm{H}, \mathrm{Ru}-\mathrm{H}-\mathrm{Ru}) ;{ }^{13} \mathrm{C}\left\{{ }^{1} \mathrm{H}\right\}$ NMR $\left(125 \mathrm{MHz}, \mathrm{CDCl}_{3}, 22{ }^{\circ} \mathrm{C}\right): \delta=93.3\left(\mathrm{~s}, C_{5} \mathrm{Me}_{5}\right), 14.2 \mathrm{ppm}$ $\left(\mathrm{s}, \mathrm{C}_{5} M e_{5}\right) ; \mathrm{IR}\left(\mathrm{DCM}, \mathrm{cm}^{-1}\right): 2914.9\left(\mathrm{Cp}^{*}\right), 2847.3(\mathrm{C}-\mathrm{H})$.

5: MS $\left(\mathrm{ESI}^{+}\right): \mathrm{m} / \mathrm{z}$ calculated for $\left[\mathrm{C}_{26} \mathrm{H}_{41} \mathrm{~B}_{4} \mathrm{SClRu}_{2}+\mathrm{H}\right]^{+}$: 669.1, found $669.1 ;{ }^{11} \mathrm{~B}\left\{{ }^{1} \mathrm{H}\right\}$ NMR $(160$ $\mathrm{MHz}, \mathrm{CDCl}_{3}, 22{ }^{\circ} \mathrm{C}$ ): $\delta=133.8$ (s, 1B), 23.0 (s, 1B), 19.9 (s, 1B), -7.9 ppm (s, 1B); ${ }^{1} \mathrm{H}$ NMR (500 $\left.\mathrm{MHz}, \mathrm{CDCl}_{3}, 22{ }^{\circ} \mathrm{C}\right): \delta=10.75\left(\mathrm{br}, \mathrm{BH}_{t}\right), 7.49-7.09(5 \mathrm{H}, \mathrm{Ph}), 4.88\left(\mathrm{br}, \mathrm{BH}_{t}\right), 1.89$ (s, $\left.15 \mathrm{H}, \mathrm{Cp}^{*}\right)$, 1.85 (s, 15H, Cp*), -9.75 (br, 1H, Ru-H-B), -9.94 (br, 1H, Ru-H-B), -11.03 (br, 1H, B-H-B), -15.66 ppm (s, 1H, Ru-H-Ru); ${ }^{13} \mathrm{C}\left\{{ }^{1} \mathrm{H}\right\}$ NMR $\left(125 \mathrm{MHz}, \mathrm{CDCl}_{3}, 22{ }^{\circ} \mathrm{C}\right): \delta=138.9-131.6(\mathrm{~s}, \mathrm{Ph}), 95.3(\mathrm{~s}$, 
$\left.C_{5} \mathrm{Me}_{5}\right), 89.0$ (s, $\left.C_{5} \mathrm{Me}_{5}\right), 22.9\left(\mathrm{~s}, \mathrm{C}_{5} M e_{5}\right), 14.3\left\{{ }^{1} \mathrm{H}\right\}\left(\mathrm{s}, \mathrm{C}_{5} M e_{5}\right) ; \mathrm{IR}\left(\mathrm{DCM}, \mathrm{cm}^{-1}\right): 2841.6(\mathrm{C}-\mathrm{H})$, $2914.9\left(\mathrm{Cp}^{*}\right)$.

\subsection{Synthesis of compound $\mathbf{6}$}

In a flame-dried Schlenk tube, nido-1 $(0.13 \mathrm{~g}, 0.25 \mathrm{mmol})$ was suspended in toluene $(5 \mathrm{~mL})$ and the mixture was cooled to $-78^{\circ} \mathrm{C}$. $\mathrm{Li}\left[\mathrm{BH}_{3}(\mathrm{SePh})\right](0.130 \mathrm{~g}, 1.0 \mathrm{mmol})$ in toluene $(12 \mathrm{~mL})$ was added via cannula and then the solution was allowed to warm slowly to room temperature. The resulting reaction mixture was then heated to $80^{\circ} \mathrm{C}$ for 18 hours. The solvent was dried and the residue was extracted into n-hexane and passed through Celite. After removal of the solvent, the residue was subjected to chromatographic work-up by using TLC plates (n-hexane/ $\mathrm{CH}_{2} \mathrm{Cl}_{2}, 85: 15 \mathrm{v} / \mathrm{v}$ ) yielded orange $\left[(\mathrm{Cp} * \mathrm{Ru})_{2} \mathrm{~B}_{4} \mathrm{H}_{9}(\mathrm{SePh})\right], \mathbf{6}(0.036 \mathrm{~g}, 20.8 \%)$ along with orange $\left[(\mathrm{Cp} * \mathrm{Ru})_{2} \mathrm{~B}_{4} \mathrm{H}_{7}(\mathrm{Ph})\right], \mathbf{3}(0.018$ $\mathrm{g}, 11.8 \%)$.

6: HR-MS $\left(\mathrm{ESI}^{+}\right): \mathrm{m} / \mathrm{z}$ calculated for $\left[\mathrm{C}_{26} \mathrm{H}_{44} \mathrm{~B}_{4} \mathrm{SeRu}_{2}+\mathrm{Na}\right]^{+}: 707.0965$ found $707.0981 ;{ }^{11} \mathrm{~B}\left\{{ }^{1} \mathrm{H}\right\}$ NMR (160 MHz, $\left.\mathrm{CDCl}_{3}, 22{ }^{\circ} \mathrm{C}\right): \delta=20.3$ (s, 1B), 19.5 (s, 1B), 4.9 (s, 2B); ${ }^{1} \mathrm{H}$ NMR $(500 \mathrm{MHz}$, $\left.\mathrm{CDCl}_{3}, 22{ }^{\circ} \mathrm{C}\right): \delta=7.02-7.15(5 \mathrm{H}, \mathrm{Ph}), 4.48\left(\mathrm{br}, \mathrm{BH}_{t}\right), 3.17\left(\mathrm{br}, \mathrm{BH}_{t}\right), 2.70\left(\mathrm{br}, \mathrm{BH}_{t}\right), 1.88(\mathrm{~s}, 15 \mathrm{H}$, Cp*), 1.76 (s, 15H, Cp*), -2.64 (br, 1H, B-H-B), -3.36 (br, 1H, B-H-B), -4.29 (br, 1H, B-H-B), 12.00 (br, 1H, Ru-H-B), -13.35 (br, 1H, Ru-H-B), -13.65 ppm (s, 1H, Ru-H-Ru); ${ }^{1} \mathrm{H}\left\{{ }^{11} \mathrm{~B}\right\}$ NMR $\left(500 \mathrm{MHz}, \mathrm{CDCl}_{3}, 22^{\circ} \mathrm{C}\right): \delta=7.04-7.48(5 \mathrm{H}, \mathrm{Ph}), 4.60\left(\mathrm{~s}, \mathrm{BH}_{t}\right), 3.02\left(\mathrm{~s}, \mathrm{BH}_{t}\right), 2.51\left(\mathrm{~s}, \mathrm{BH}_{t}\right), 1.89(\mathrm{~s}$, 15H, Cp*), 1.77 (s, 15H, Cp*), -2.64 (s, 1H, B-H-B), -3.37 (s, 1H, B-H-B), -4.29 (s, 1H, B-H-B), 11.92 (s, 1H, Ru-H-B), -13.35 (s, 1H, Ru-H-B), -13.65 ppm (s, 1H, Ru-H-Ru); ${ }^{13} \mathrm{C}\left\{{ }^{1} \mathrm{H}\right\}$ NMR (125 $\mathrm{MHz}, \mathrm{CDCl}_{3}, 22^{\circ} \mathrm{C}$ ): $\delta=136.7$ (s, quaternary carbon), 131.0-124.1 (s, Ph), $97.6\left(\mathrm{~s}, C_{5} \mathrm{Me}_{5}\right), 89.9$ (s, $C_{5} \mathrm{Me}_{5}$ ), 11.5 (s, $\left.\mathrm{C}_{5} M e_{5}\right), 10.6$ ppm (s, $\mathrm{C}_{5} \mathrm{Me}_{5}$ ); IR (DCM, cm ${ }^{-1}$ ): 796.5 (Ru-Se), $2852.7(\mathrm{C}-\mathrm{H})$, $2915.8\left(\mathrm{Cp}^{*}\right), 2965.0\left(\mathrm{Cp}^{*}\right)$.

\subsection{Synthesis of compound 7}

In a flame-dried Schlenk tube, nido-1 $(0.13 \mathrm{~g}, 0.25 \mathrm{mmol})$ was suspended in toluene $(5 \mathrm{~mL})$ and the mixture was cooled to $-78^{\circ} \mathrm{C}$. $\mathrm{Li}\left[\mathrm{BH}_{3}(\mathrm{TePh})\right](0.225 \mathrm{~g}, 1.0 \mathrm{mmol})$ in toluene $(12 \mathrm{~mL})$ was added 


\section{ACCEPTED MANUSCRIPT}

via cannula and then the solution was allowed to warm slowly to room temperature. The resulting reaction mixture was then heated to $80{ }^{\circ} \mathrm{C}$ for 18 hours. The solvent was dried and the residue was extracted into n-hexane and passed through Celite. After removal of the solvent, the residue was subjected to chromatographic work-up by using TLC plates ( $n$-hexane/ $\mathrm{CH}_{2} \mathrm{Cl}_{2}, 85: 15 \mathrm{v} / \mathrm{v}$ ) yielded pink [(Cp*Ru $\left.)_{2} \mathrm{~B}_{4} \mathrm{H}_{6} \mathrm{Te}\right], 7(0.031 \mathrm{~g}, 18.8 \%)$ along with the orange compound [(Cp* Ru $\left.)_{2} \mathrm{~B}_{4} \mathrm{H}_{7}(\mathrm{Ph})\right]$, $3(0.009 \mathrm{~g}, 5.9 \%)$

7: HR-MS $\left(\mathrm{ESI}^{+}\right): \mathrm{m} / z$ calculated for $\left[\mathrm{C}_{20} \mathrm{H}_{36} \mathrm{Ru}_{2} \mathrm{~B}_{4} \mathrm{Te}+\mathrm{H}\right]^{+}: 655.0416$, found $655.0420 ;{ }^{11} \mathrm{~B}\left\{{ }^{1} \mathrm{H}\right\}$ $\operatorname{NMR}\left(160 \mathrm{MHz}, \mathrm{CDCl}_{3}, 22{ }^{\circ} \mathrm{C}\right): \delta=80.3$ (s, 1B), 23.1 (s, 1B), 17.5 (s, 1B), -17.5 ppm (s, 1B); ${ }^{1} \mathrm{H}$ $\operatorname{NMR}\left(500 \mathrm{MHz}, \mathrm{CDCl}_{3}, 22{ }^{\circ} \mathrm{C}\right): \delta=6.51\left(\mathrm{br}, \mathrm{BH}_{t}\right), 4.92\left(\mathrm{br}, \mathrm{BH}_{t}\right), 4.19\left(\mathrm{br}, \mathrm{BH}_{t}\right),-0.21\left(\mathrm{br}, \mathrm{BH}_{t}\right)$, 1.92 (s, 15H, Cp*), 1.69 (s, 15H, Cp*), -0.21 (br, BH ( $^{*},-4.23$ (br, 1H, B-H-B), -12.42 ppm (br, 1H, $\mathrm{B}-\mathrm{H}-\mathrm{B}) ;{ }^{1} \mathrm{H}\left\{{ }^{11} \mathrm{~B}\right\} \mathrm{NMR}\left(500 \mathrm{MHz}, \mathrm{CDCl}_{3}, 22{ }^{\circ} \mathrm{C}\right): \delta=6.70\left(\mathrm{br}, \mathrm{BH}_{t}\right), 5.17\left(\mathrm{br}, \mathrm{BH}_{t}\right), 4.15\left(\mathrm{~s}, \mathrm{BH}_{t}\right),-$ $0.18\left(\mathrm{~s}, \mathrm{BH}_{t}\right), 1.92\left(\mathrm{~s}, 15 \mathrm{H}, \mathrm{Cp}^{*}\right), 1.69$ (s, 15H, Cp*), -4.24 (s, 1H, B-H-B), -12.51 ppm (s, 1H, B-H$\mathrm{B}) ;{ }^{13} \mathrm{C}\left\{{ }^{1} \mathrm{H}\right\} \mathrm{NMR}\left(125 \mathrm{MHz}, \mathrm{CDCl}_{3}, 22{ }^{\circ} \mathrm{C}\right): \delta=94.9\left(\mathrm{~s}, C_{5} \mathrm{Me}_{5}\right), 93.4\left(\mathrm{~s}, C_{5} \mathrm{Me}_{5}\right), 12.6\left(\mathrm{~s}, \mathrm{C}_{5} M e_{5}\right)$, 11.5 ppm (s, $\left.\mathrm{C}_{5} \mathrm{Me}_{5}\right)$; IR (DCM, cm $\left.{ }^{-1}\right): 2922.6\left(\mathrm{Cp}^{*}\right), 2849.3(\mathrm{C}-\mathrm{H})$.

\subsection{X-ray crystal structure determinations}

\section{(Table 4 near here)}

\subsection{Computational details}

Quantum chemical calculations were performed on compound 2' ( $\mathrm{Cp}$ analogue of 2) using density functional theory (DFT) as implemented in the Gaussin09 package [27]. The calculations were carried out on the $\mathrm{Cp}$ analogue in order to reduce computational effort. The geometry optimization was carried out without any symmetry constraints, in gaseous state (no solvent effect) using the PBE0 functional [28] with the triple- $\zeta$ quality basis set Def2-TZVP. The projected ${ }^{11} \mathrm{~B}$ chemical shielding values, computed at the PBE0/Def2-TZVP level of theory, were referenced to $\mathrm{B}_{2} \mathrm{H}_{6}$ (B shielding constant $85.9 \mathrm{ppm}$ ), and were then converted to the standard $\mathrm{BF}_{3} \cdot \mathrm{OEt}_{2}$ scale using the experimental value of $+16.6 \mathrm{ppm}$ for $\mathrm{B}_{2} \mathrm{H}_{6} .{ }^{1} \mathrm{H}$ chemical shifts were referenced to TMS ( $\mathrm{SiMe}_{4}$ ). 
Computation of the NMR shielding tensors employed gauge-including atomic orbitals (GIAOs) [29], using the implementation of Schreckenbach, Wolff, Ziegler, and co-workers [30]. Natural bond orbital (NBO) analysis [31] within the Gaussian09 package was carried out at the same level of theory. Wiberg bond indexes (WBI) [32] values on some selected bonds were obtained from a natural bond orbital (NBO) analysis. The ChemCraft package [33] was used for the visualizations. Laplacian maps of the electron density distribution plots were generated using the Multiwfn package [34].

\section{Conflict of interest}

The authors declare no competing financial interest.

\section{Acknowledgments}

This work was supported by the Council of Scientific and Industrial Research (CSIR; No. 01(2837)/15/EMR-II) New Delhi, India. B. J. is grateful to University Grants Commission (UGC) for research fellowship. S. G. and S. K. B. are grateful to IIT Madras for research fellowships. We thank R. Ramalakshmi (IIT Madras) for helpful discussions.

\section{Appendix A. Supplementary data}

Supplementary data related to this article can be found at http://dx.doi.org/

\section{References}

[1] (a) J. D. Kennedy, The Polyhedral Metallaboranes Part II. Metallaborane Clusters with Eight Vertices and More. pp. 519-679. In S. J. Lippard (ed.) Progress in Inorganic Chemistry, John Wiley and Sons, New York, (1986);

(b) R. N. Grimes, Coord. Chem. Rev. 200-202 (2000) 773-811.

(c) R. N. Grimes, Carboranes, Academic Press, Elsevier, Oxford, UK, 2nd edn, (2011).

[2] (a) G. D. Friesen, A. Barriola, P. Daluga, P. Ragatz,J. C. Huffman, L. J. Todd, Inorg. Chem.19 (1980) 458-462;

(b) N. C. Norman, A. G. Orpen, M. J. Quayle, C. R. Rice, New J. Chem. 24 (2000) 837-839; 
(c) A. Hammerschmidt, M. Döch, S. Pütz, B. Krebs, Z. Anorg. Allg. Chem. 631 (2005) 11251329 ;

(d) B. Frange, J. D. Kennedy, Main Group Met. Chem. 19 (1996) 1775-1777;

(e) G. Ferguson, J. D. Kennedy,X. L. R. Fontaine, Faridoon, T. R. Spalding, J. Chem. Soc., Dalton Trans.(1988) 2555-2564;

(f) G. Ferguson, J. F. Gallagher, J. D. Kennedy, A.-M. Kelleher, T. R. Spalding, Dalton Trans. (2006) 2133-2139;

(g) S. K. Bose, S. M. Mobin, S. Ghosh, J. Organomet. Chem. 696 (2011) 3121-3126.

[3] (a) Faridoon, M. McGrath, T. R. Spalding, X. L. R. Fontaine, J. D. Kennedy, M. T. Pett,J. Chem. C. Dalton Trans. (1990) 1819-1829.

(b) K. Geetharani, S. K. Bose, S. Sahoo, S. Ghosh, Angew. Chem. Int. Ed. 50 (2011) 39083911 ;

(c) S. K. Barik, M. G. Chowdhury, S. De, P. Parameswaran, S. Ghosh, Eur. J. Inorg. Chem. (2016) 4546-4550.

(d) K. K. V. Chakrahari, A. Thakur, B. Mondal, V. Ramkumar, S. Ghosh, Inorg. Chem. 52 (2013) 7923-2932;

(e) D. K. Roy, S. K. Bose, K. Geetharani, K. K. V. Chakrahari, S. M. Mobin, S. Ghosh, Chem. Eur. J. 18 (2012) 9983-9991.

[4] (a) X. Meng, A. K. Bandyopadhyay, T. P. Fehlner, F.-W. Grevels, J. Organomet. Chem. 394 (1990) 15-27.

[5] (a) S. K. Bose, K. Geetharani, V. Ramkumar, B. Varghese, S. Ghosh, Inorg. Chem. 49 (2010) 2881-2888;

(b) S. Ghosh, B. C. Noll, T. P. Fehlner, Angew. Chem. Int. Ed. 44 (2005) 2916-2918.

[6] (a) T. P. Fehlner, J. Chem. Soc. Dalton Trans. (1998) 1525-1532;

(b) T. P. Fehlner, Organometallics 19 (2000) 2643-2651;

(c) S. Ghosh, A. L. Rheingold, T. P. Fehlner, Chem. Commun. (2001) 895-896.

[7] (a) A. Burke, D. Ellis, B. T. Giles, B. E. Hodson, S. A. Macgregor, G. M. Rosair, A. J. Welch, Angew. Chem. Int. Ed. 42 (2003) 225-228;

(b) A. Burke, D. Ellis, D. Ferrer, D. L. Ormbsy, G. M. Rosair, A. J. Welch, Dalton. Trans. (2005) 1716-1721.

[8] (a) L. Deng, J. Zhang, H.-S. Chan, Z. Xie, Angew. Chem. Int. Ed. 45 (2006) 4309-4313;

(b) L. Deng, Z. Xie, Coord. Chem. Rev. 251 (2007) 2452-2476;

(c) J. Zhang, H.-S. Chan, Z. Xie, Angew. Chem. Int. Ed.47 (2008) 9447-9449. 
[9] (a) D. K. Roy, S. K. Bose, R. S. Anju, B. Mondal, V. Ramkumar, S. Ghosh, Angew. Chem. Int. Ed. 52 (2013) 3222-3226;

(b) D. K. Roy, B. Mondal, P. Shankhari, R. S. Anju, K. Geetharani, S. M. Mobin, S. Ghosh, Inorg. Chem. 52 (2013) 6705-6712;

(c) D. K. Roy, S. Ghosh, J.-F. Halet, J. Cluster Sci. 25 (2014) 225-237.

[10] (a) P. Shankhari, D. K. Roy, K. Geetharani, R.S. Anju, B. Varghese, S. Ghosh, J. Organomet. Chem. 747 (2013) 249-253;

[11] S. Sahoo, S. M. Mobin, S. Ghosh, J. Organomet. Chem.695 (2010) 945-949.

[12] S. K. Barik, V. Dorcet, T. Roisnel, J.-F.Halet, S. Ghosh, Dalton Trans. 44 (2015), 1440314410.

[13] (a) X. Lei, M. Shang, T.P. Fehlner, J. Am. Chem. Soc.121 (1999) 1275-1287.

(b) S. Ghosh, B. C. Noll, T. P. Fehlner, Angew. Chem. Int. Ed. 44 (2005) 6568-6571;

(c) R. S. Anju, D. K. Roy, B. Mondal, K. Yuvaraj, C. Arivazhagan, K. Saha, B. Varghese, S. Ghosh, Angew. Chem. Int. Ed. 53 (2014) 2873-2877;

(d) D. K. Roy, B. Mondal, R. S. Anju, S. Ghosh, Chem. Eur. J. 21 (2015) 3640-3648.

[14] (a) A. D. Pasquale, X. Lei, T. P. Fehlner, Organometallics 20 (2001) 5044-5049;

(b) R. S. Anju, K. Saha, B. Mondal, V. Dorcet, T. Roisnel, J.-F.Halet, S. Ghosh, Inorg. Chem. 53 (2014) 10527-10535.

[15] K. Wade, Inorg. Nucl. Chem. Lett. 8 (1972) 559-562.

[16] (a) D.M.P. Mingos, Nature. Phys. Sci. 236 (1972) 99-102;

(b) D.M.P. Mingos, Acc. Chem. Res. 17 (1984) 311-319.

[17] The topological parameters calculated for the B10-B11-B12-Ru2-S9 motif, i.e., the electron density $\rho$ at the bond critical points (BCPs $(3,-1)$ ) and the Laplacian of the density $\nabla^{2} \rho$, are listed in Table $\mathrm{S} 3$.

[18] X. Lei, M. Shang, T.P. Fehlner, Inorg. Chem. 37 (1998) 3900-3901.

[19] X. Lei, M. Shang, T. P. Fehlner, Organometallics 19 (2000) 118-120.

[20] (a) G. D. Friesen, A. Barriola, P. Daluga, P. Ragatz, J.C. Huffman, L. J. Todd, Inorg. Chem. 19 (1980) 458-462;

(b) N.C. Norman, A.G. Orpen, M.J. Quayle, C.R. Rice, New J. Chem. 24 (2000) 837-841;

(c) A. Hammerschmidt, M. Döch, S. Pütz , B. Krebs, Z. Anorg. Allg. Chem. 631 (2005) 11251128.

[21] J. Bould, N.P. Rath, L. Barton, Organometallics 14 (1995) 2119-2122.

[22] U. Koelle, J. Kossakowski, Inorg. Synth. 29 (1992) 225-228. 
[23] R. Ramalakshmi, K. Saha, D.K. Roy, B. Varghese, A.K. Phukan, S. Ghosh, Chem. Eur. J. 21 (2015) 17191-17195.

[24] G. E. Ryschkewitsch, K. C. Nainan, Inorg. Synth. 15 (1974) 111-118.

[25] (a) A. Altornare, G. Cascarano, C. Giacovazzo, A. Guagliardi, J. Appl. Cryst. 26 (1993) 343350 ;

(b) G. M. Sheldrick, Acta Cryst. A71 (2015) 3-8.

[26] G. M. Sheldrick, Acta Cryst. C71 (2015) 3-8.

[27] M. J. Frisch, G. W. Trucks, H. B. Schlegel, G. E. Scuseria, M. A. Robb, J. R. Cheeseman, G. Scalmani, V. Barone, B. Mennucci, G. A. Petersson, H. Nakatsuji, M. Caricato, X. Li, H. P.Hratchian, A. F. Izmaylov, J. Bloino, G. Zheng, J. L. Sonnenberg, M. Hada, M. Ehara, K. Toyota, R. Fukuda, J. Hasegawa, M. Ishida, T. Nakajima, Y. Honda, O. Kitao, H. Nakai, T. Vreven, J. A. Jr. Montgomery, J. E. Peralta, F. Ogliaro, M. Bearpark, J. J. Heyd, E. Brothers, K. N. Kudin, V. N. Staroverov, T. Keith, R. Kobayashi, J. Normand, K. Raghavachari, A. Rendell, J. C. Burant, S. S. Iyengar, J. Tomasi, M. Cossi, N. Rega, J. M Millam, M. Klene, J. E. Knox, J. B. Cross, V.Bakken, C. Adamo, J. Jaramillo, R. Gomperts, R. E Stratmann, OYazyev, A. J Austin, R. Cammi, C. Pomelli, J. W. Ochterski, R. L. Martin, K. Morokuma, V. G. Zakrzewski, G. A. Voth, P. Salvador, J. J. Dannenberg, S. Dapprich, A. D. Daniels, O. Farkas, J. B. Foresman, J. V. Ortiz, J. Cioslowski, D. J. Fox, Gaussian 09, Revision C.01; Gaussian, Inc.: Wallingford, CT(2010).

[28] J. P. Perdew, K. Burke, M. Ernzerhof, Phys. Rev. Lett. 77 (1996) 3865-3868.

[29] (a) F. London, J. Phys. Radium. 8 (1937) 397-409.

(b) R. Ditchfield, Mol. Phys. 27 (1974) 789-807.

(c) K. Wolinski, J. F. Hinton, P. Pulay, J. Am. Chem. Soc. 112 (1990) 8251-8260.

[30] (a) G. Schreckenbach, T. Ziegler, J. Phys. Chem. 99 (1995) 606-611.

(b) G. Schreckenbach, T. Ziegler, Int. J. Quantum Chem. 61 (1997) 899-918.

(c) G. Schreckenbach, T. Ziegler, Int. J. Quantum Chem. 60 (1996) 753-766.

(d) S. K. Wolff, T. Ziegler, Chem. J. Chem. Phys. 109 (1998) 895-905.

(e) S. K. Wolff, T. Ziegler, E. van Lenthe, E. J. Baerends, J. Chem. Phys. 110 (1999) 76897698.

[31] (a) F. Weinhold, C. R. Landis, Valency and bonding: A natural bond orbital donor acceptor perspective; Cambridge University Press: Cambridge, U.K, (2005).

(b) A. E. Reed, L. A. Curtiss, F. Weinhold, Chem. Rev. 88 (1988) 899-926.

[32] K.Wiberg, Tetrahedron, 24 (1968) 1083-1096.

[33] G. A. Zhurko, http://www.chemcraftprog.com. 
ACCEPTED MANUSCRIPT

[34] T. Lu,F. Chen, J. Comput. Chem. 33 (2012) 580-592. 


\section{ACCEPTED MANUSCRIPT}

\section{Table of Content}

Reactivity of diruthenaborane analogue of pentaborane (9) nido-[1,2-(Cp* RuH $\left.{ }_{2} \mathrm{~B}_{3} \mathrm{H}_{7}\right]$, 1, with phenyl-chalcogenoborates $\mathrm{Li}\left[\mathrm{BH}_{3}(\mathrm{EPh})\right](\mathrm{E}=\mathrm{S}, \mathrm{Se}$ or Te$)$.

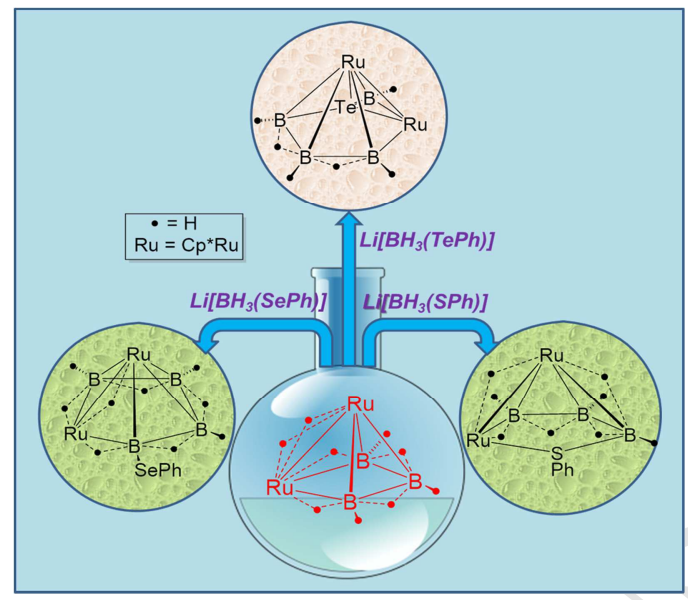

Scheme 1. Synthesis of ruthenaheteroboranes and ruthenaboranes (2-7).

Fig. 1. Molecular structure of compound 2. Selected interatomic distances $(\AA)$ and angles ( $\left.{ }^{\circ}\right)$ : Ru1-Ru2 2.9750(6), Ru1-B10 2.210(7), Ru1-B12 2.176(8), Ru1-B11 2.135(9), Ru2-S9 2.3864(15), B10-B11 1.827(12), B11-B12 1.867(10), B10-S9 1.940(7); B10-Ru1-Ru2 76.86(18), B12-Ru1-Ru2 49.94(19), B12-Ru1-B10 82.7(3), B10-S9-Ru2 98.0(2).

Table 1. Selected structural parameters and ${ }^{11} \mathrm{~B}\left\{{ }^{1} \mathrm{H}\right\}$ chemical shifts of some reported dimetalla analogues of pentaborane(11).

Fig. 2. Laplacian maps of the electron density distribution, $\nabla^{2} \rho(\mathrm{r})$, in the Ru2-S9-B10 (a) and B10-B11-B12 (b) planes of 2' (Cp model analogue of 2), Gray lines indicate areas of charge depletion $\left(-\nabla^{2} \rho(\mathrm{r})<0\right)$ and red lines indicate areas of charge concentration $(-$ $\left.\nabla^{2} \rho(\mathrm{r})>0\right)$. Solid brown lines and dotted blue lines represent bond paths and bond critical points (BCPs), respectively. Selected frontier molecular orbitals of $\mathbf{2}^{\prime}$ (isocontour values: $\left.\pm 0.052\left(\mathrm{e} \mathrm{bohr}^{-3}\right)^{1 / 2}\right)$ (c-e).

Fig. 3. Molecular structure of compounds 3 (a), 4 (b), and 5 (c). The Cp* ligand attached to $\mathrm{Ru} 1$ in $\mathbf{1}$ is omitted for clarity. Some of the bridging $\mathrm{B}-\mathrm{H}-\mathrm{B}, \mathrm{Ru}-\mathrm{H}-\mathrm{B}$ and $\mathrm{Ru}-\mathrm{H}-\mathrm{Ru}$ 
hydrogen atoms were not located crystallographically in 5. Selected interatomic distances $(\AA)$ and angles $\left(^{\circ}\right), 3$ : B1-B2 1.750(5), B1-Ru2 2.043(3), Ru1-Ru2 2.8580(3), B3-C25 2.8580(3), B2-B1-Ru2 71.96(16), B4-B2-B3 64.2(2); 4: B21-B22 1.816(4), B22-C11 1.859(3), Ru1-B21 2.029(3), Ru1-Ru2 2.8899(3); B21-Ru1-B22 50.27(11), B21-Ru1-B23 96.08(11); 5: Ru1-B1 2.043(7), Ru1-Ru2 2.8744(10), Ru2B1 2.042(7), B2-Cl1 1.871(7), B4-S21 1.911(7), B2-B4 1.746(9), B2-B3 1.755(10), B3-Ru1-B2 46.5(3), B1-Ru1-Ru2 45.27(19), B2-Ru1-Ru2 49.74(17), B1-Ru2-B2 $50.1(3)$.

Table 2. Comparison of structural parameters and ${ }^{11} \mathrm{~B}\left\{{ }^{1} \mathrm{H}\right\}$ NMR chemical shifts of 3-5 with other related compounds.

Fig. 4. Molecular structure of compound 6. The phenyl group attached to the Se1 atom is omitted for clarity. Selected interatomic distances $(\AA)$ and angles $\left(^{\circ}\right)$ : Ru1-B21 2.167(3), Ru1-B22 2.126(4), Ru1-B23 2.130(4), Ru1-B24 2.164(3), Ru1-Ru2 2.8633(4), Ru2-B21 2.282(3), Ru2-B24 2.343(3), B24-Se1 2.017(4), B21-B22 1.801(5), B22-B23 1.817(5), B23-B24 1.806(5), B22-Ru1-Ru2 89.48(9), B23-Ru1Ru2 89.75(9), B24-Ru1-Ru2 53.39(9).

Table 3. Comparison of structural parameters and ${ }^{11} \mathrm{~B}\left\{{ }^{1} \mathrm{H}\right\}$ NMR chemical shifts of 6 and other related compounds.

Fig. 5. Molecular structure of compound 7. Some of the terminal B-H and bridging B-H-B, $\mathrm{Ru}-\mathrm{H}-\mathrm{B}, \mathrm{Ru}-\mathrm{H}-\mathrm{Ru}$ hydrogen atoms were not crystallographically located. Selected interatomic distances $(\AA)$ and angles $\left(^{\circ}\right)$ : B1-B6 1.70(4), B1-B2 1.759(11), B1-Ru2 2.245(17), B1-Te1 2.23(2), B2-Ru2 2.20(2), B4-Ru1 2.25(3), B6-Ru1 2.38(3), Ru1Te1 2.532(3), Ru1-Ru2 2.8556(5), B4-Ru2 2.22(3), B6-Ru2 2.31(3), Ru2-Te1 2.584(3), B6-B1-B2 109.0(2), B6-B1-Ru2 70.2(12), B2-B1-Ru2 65.5(11), B1-B2-Te1 121.0(17), Te1-B2-Ru2 72.1(7), B4-Ru1-Ru2 49.9(8), B6-Ru1-Ru2 51.5(7), Te1Ru1-Ru2 56.95(6).

Table 4. X-ray crystal structure determinations 
Scheme 1.

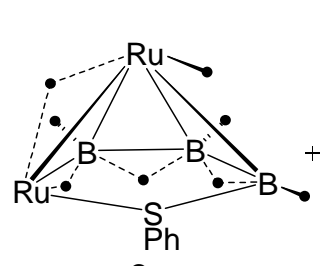

2

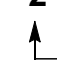

$\uparrow$

$$
\begin{gathered}
\bullet=\mathrm{H} \\
\mathrm{Ru}=\mathrm{Cp}{ }^{\star} \mathrm{Ru}
\end{gathered}
$$

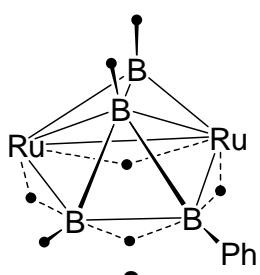

3

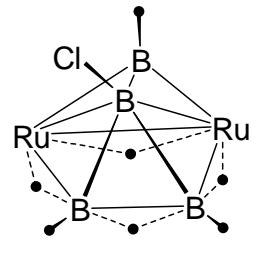

4

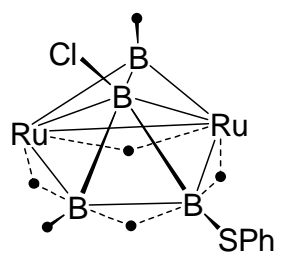

5

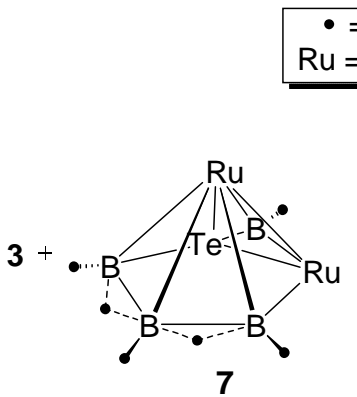

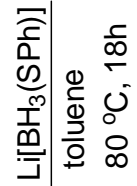
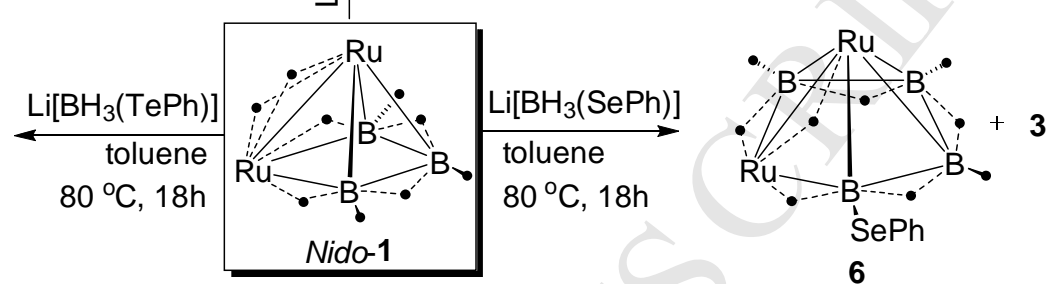

Fig. 1.

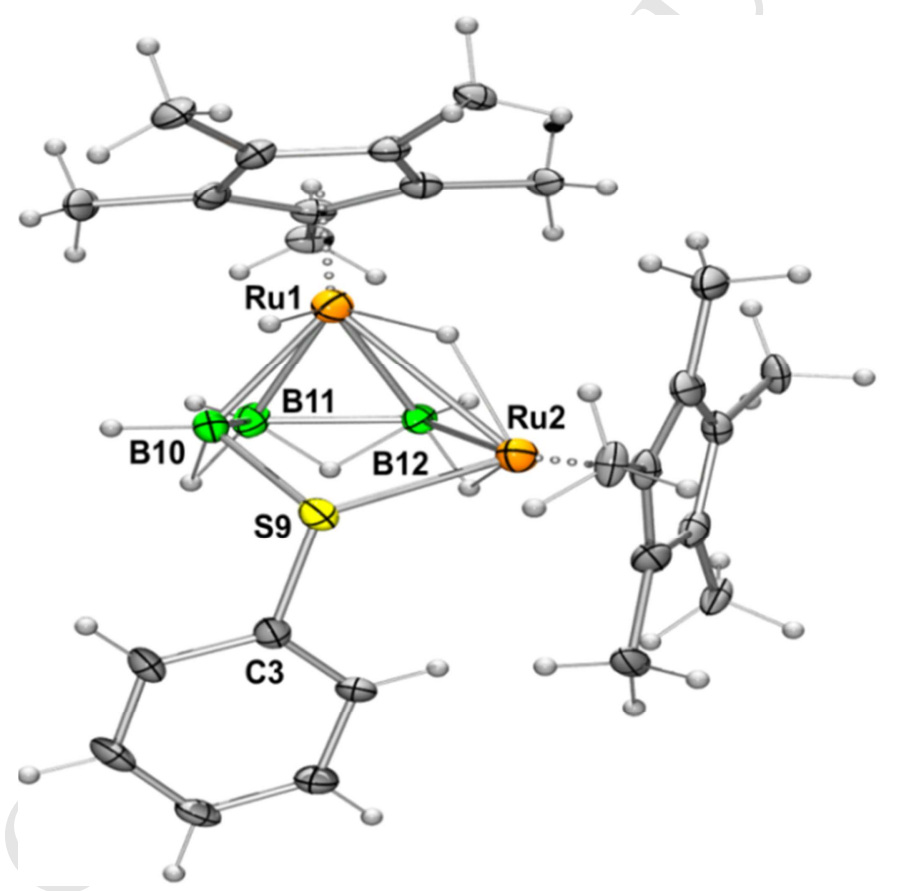


Table 1.

Compound $^{\mathrm{a}} \quad d_{\mathrm{M}-\mathrm{M}}[\AA] \quad d_{\mathrm{B}-\mathrm{B}}[\AA]^{\mathrm{b}} \quad{ }^{\mathrm{II}} \mathrm{B}\left\{{ }^{\mathrm{I}} \mathrm{H}\right\}$ NMR $\delta[\mathrm{ppm}] \quad$ Ref.

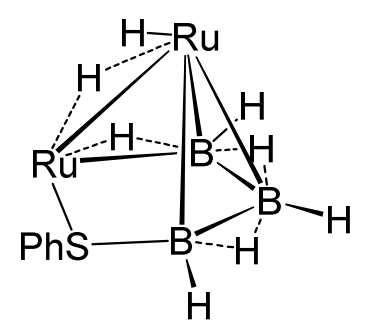

2.975

1.840

$30.2,4.9,-1.7$

This work

2

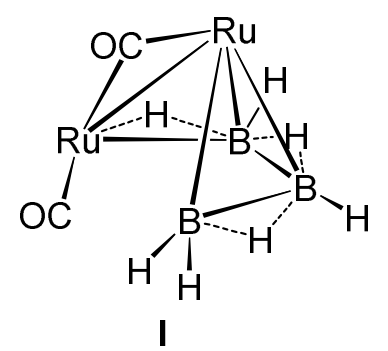

2.875

1.801

$36.7,29.9$

[14a]

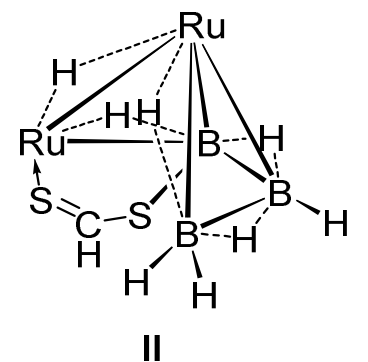

2.974

1.803

$35.6,-3.1,-13.0$

[14b]

${ }^{\mathrm{a}} \mathrm{Ru}=\mathrm{Cp} * \mathrm{Ru}$

${ }^{\mathrm{b}}$ Average B-B distance. 
Fig. 2.

a)

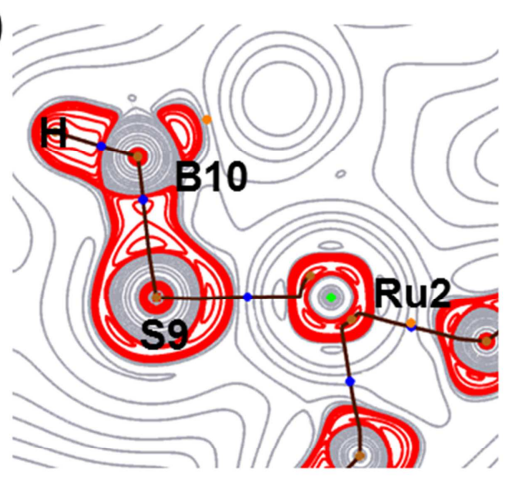

b)

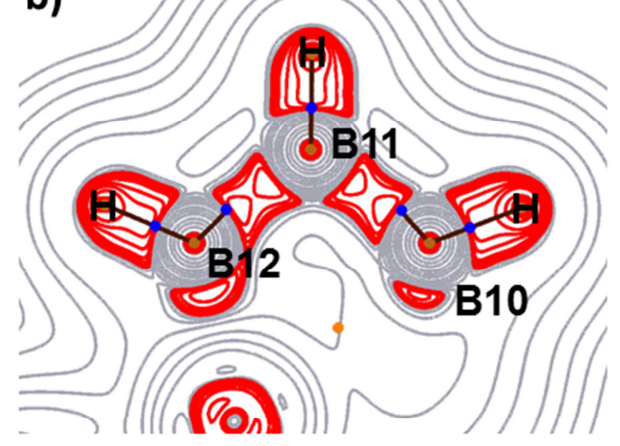

c)

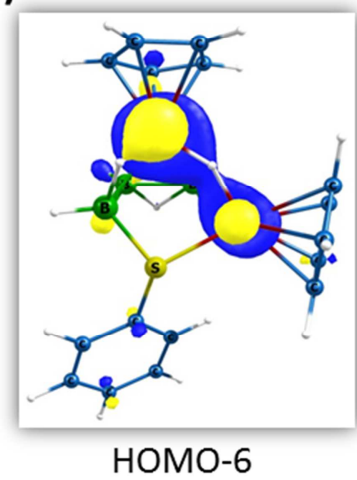

d)

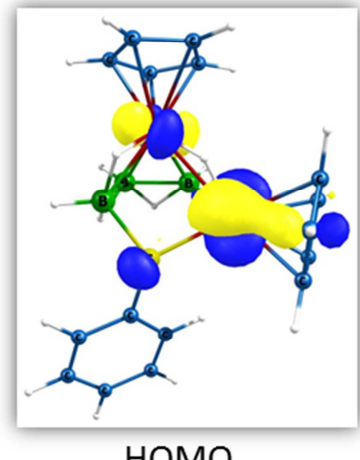

e)

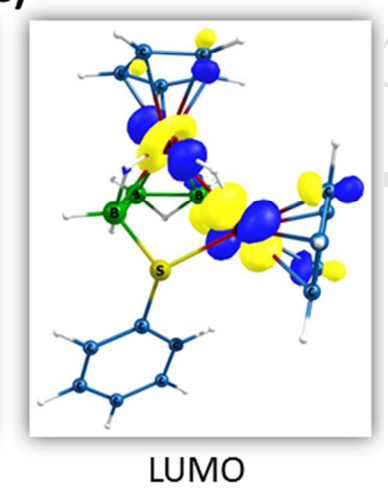


Fig. 3.

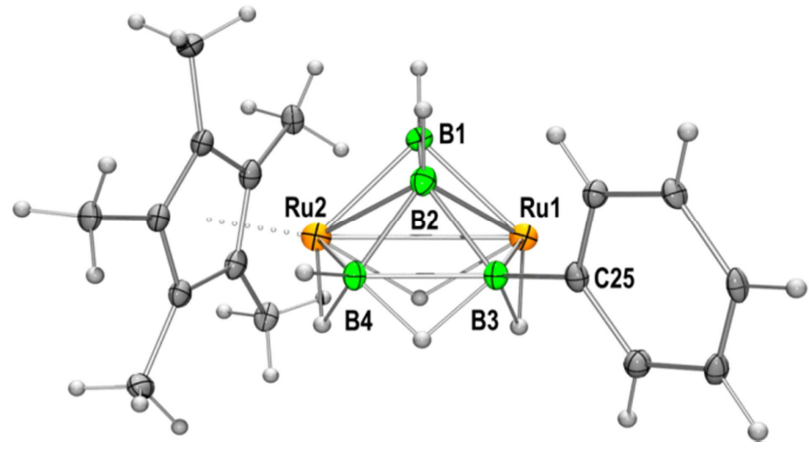

(a)

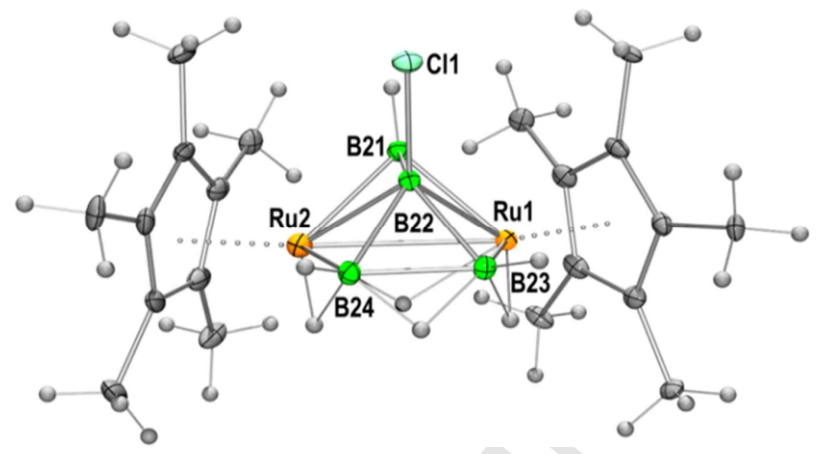

(b)

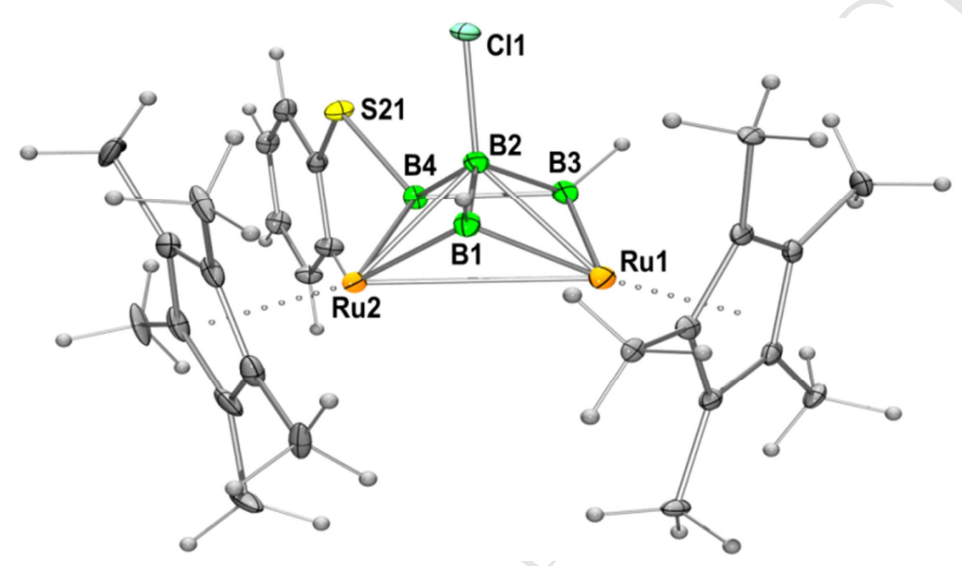

(c)

Table 2.

\begin{tabular}{lccc}
\hline Compound & $d_{\mathrm{M}-\mathrm{M}}\left[\AA{ }^{\circ}\right]^{\mathrm{a}}$ & $d_{\mathrm{B}-\mathrm{B}}[\AA]$ & ${ }^{11} \mathrm{~B}\left\{{ }^{1} \mathrm{H}\right\}$ NMR $\delta[\mathrm{ppm}]$ \\
\hline$\left[(\mathrm{Cp} * \mathrm{Ru})_{2} \mathrm{~B}_{4} \mathrm{H}_{7}(\mathrm{Ph})\right], \mathbf{3}$ & 2.858 & 1.767 & $124.7,33.8,21.5,-31.6$ \\
{$\left[(\mathrm{Cp} * \mathrm{Ru})_{2} \mathrm{~B}_{4} \mathrm{H}_{7}(\mathrm{Cl})\right], \mathbf{4}$} & 2.889 & 1.777 & $128.8,15.7,-10.8$ \\
{$\left[(\mathrm{Cp} * \mathrm{Ru})_{2} \mathrm{~B}_{4} \mathrm{H}_{6}(\mathrm{SPh})(\mathrm{Cl})\right], \mathbf{5}$} & 2.874 & 1.790 & $133.8,23.0,19.9,-7.9$ \\
{$\left[(\mathrm{Cp} * \mathrm{Ru})_{2} \mathrm{~B}_{4} \mathrm{H}_{7}(\mu-\mathrm{H})\right][13 \mathrm{a}]$} & 2.855 & 1.720 & $122.5,21.3,-32.8$ \\
{$\left[(\mathrm{Cp} * \mathrm{Ru})_{3} \mathrm{~B}_{3} \mathrm{H}_{7}(\mu-\mathrm{H})\right][18]$} & 2.806 & 1.874 & $128.3,14.4$
\end{tabular}

${ }^{a}$ Average B-B distance. 
Fig. 4.

Table 3.

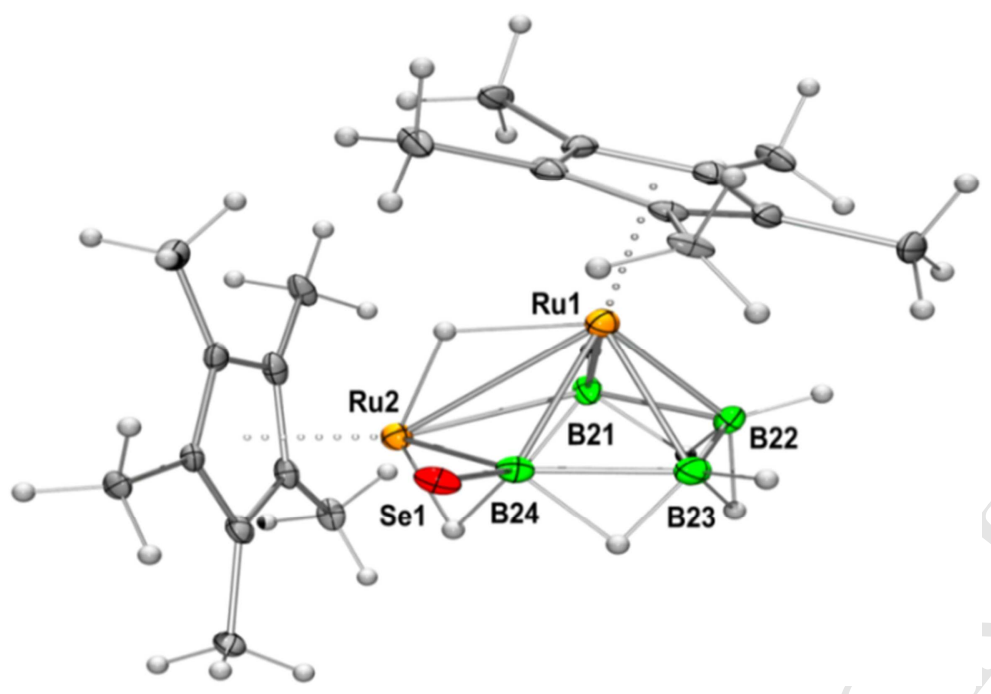

\begin{tabular}{llll}
\hline Compound & $d_{\mathrm{M}-\mathrm{M}}[\AA]$ & $d_{\mathrm{B}-\mathrm{B}}\left[\AA{ }^{\mathrm{a}}\right.$ & ${ }^{11} \mathrm{~B}\left\{{ }^{1} \mathrm{H}\right\} \mathrm{NMR} \delta[\mathrm{ppm}]$ \\
\hline$\left[(\mathrm{Cp} * \mathrm{Ru})_{2} \mathrm{~B}_{4} \mathrm{H}_{9}(\mathrm{SePh})\right], \mathbf{6}$ & 2.863 & 1.806 & $20.3,19.5,4.9$ \\
{$\left[(\mathrm{Cp} * \mathrm{Ru})_{2} \mathrm{~B}_{4} \mathrm{H}_{9}(\mu-\mathrm{H})\right][13 \mathrm{a}]$} & 2.852 & 1.831 & $17.1,3.9$ \\
{$\left[(\mathrm{Cp} * \mathrm{Ir})_{2} \mathrm{~B}_{4} \mathrm{H}_{7}(\mu-\mathrm{H})\right][19]$} & - & 1.869 & $133.8,23.0,19.9,-7.9$ \\
& & 1.869 & $133.8,23.0,19.9,-7.9$ \\
\hline$\left[(\mathrm{Cp} * \mathrm{Ir})_{2} \mathrm{~B}_{4} \mathrm{H}_{7}(\mu-\mathrm{H})\right][19]$ & - & & \\
${ }^{\mathrm{a}}$ Average B-B distance. & & &
\end{tabular}

Fig. 5.

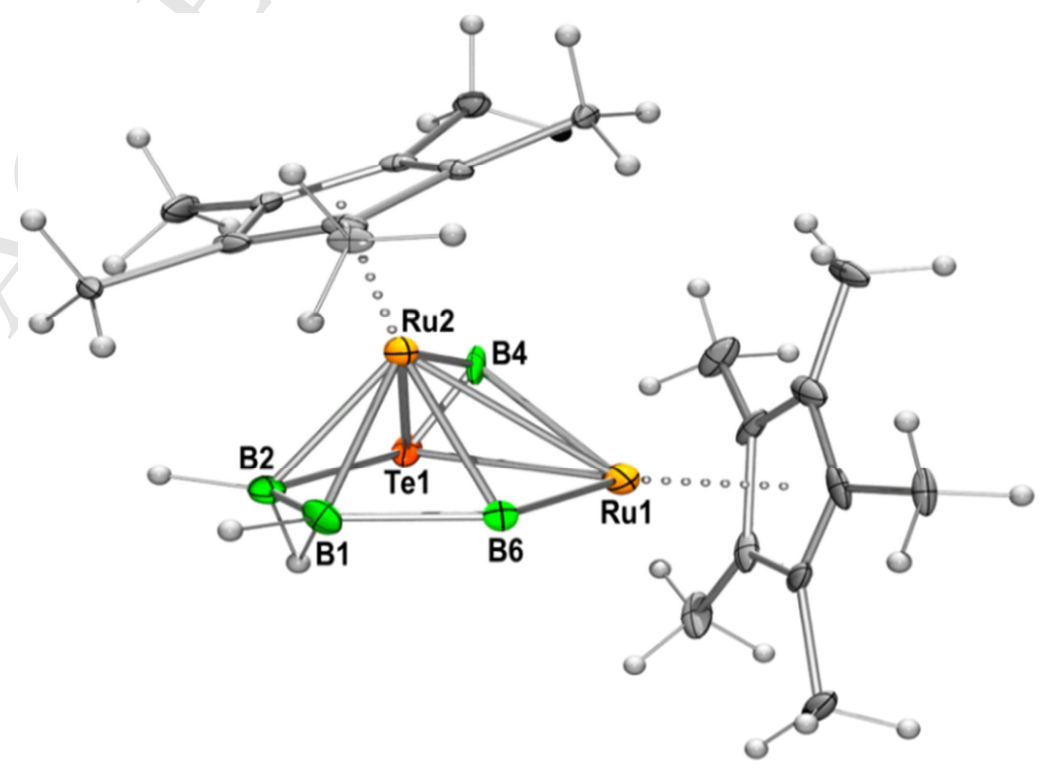


Table 4

\begin{tabular}{|c|c|c|c|c|c|c|}
\hline Compound & 2 & 3 & 4 & 5 & 6 & 7 \\
\hline CCCDC no. & 1564232 & 1564233 & 1564234 & 1564236 & 1564236 & 1564237 \\
\hline Empirical formula & $\mathrm{C}_{26} \mathrm{H}_{43} \mathrm{~B}_{3} \mathrm{SRu}_{2}$ & $\mathrm{C}_{26} \mathrm{H}_{42} \mathrm{~B}_{4} \mathrm{Ru}_{2}$ & $\mathrm{C}_{20} \mathrm{H}_{37} \mathrm{~B}_{4} \mathrm{ClRu}_{2}$ & $\mathrm{C}_{26} \mathrm{H}_{37} \mathrm{~B}_{4} \mathrm{ClRu}_{2} \mathrm{~S}$ & $\mathrm{C}_{26} \mathrm{H}_{44} \mathrm{~B}_{4} \mathrm{Ru}_{2} \mathrm{Se}$ & $\mathrm{C}_{20} \mathrm{H}_{37} \mathrm{~B}_{4} \mathrm{Ru}_{2} \mathrm{Te}$ \\
\hline Formula weight & 622.23 & 599.97 & 558.32 & 662.44 & 680.95 & 650.47 \\
\hline Crystal system & Monoclinic & Monoclinic & Triclinic & Monoclinic & Triclinic & Monoclinic \\
\hline Space group & $P 2_{1}$ & $P 2_{1} / n$ & $P-1$ & $P 2_{1} / n$ & $P-1$ & $C 2$ \\
\hline$a(\AA)$ & $9.3083(4)$ & $12.1534(3)$ & $9.0594(8)$ & $11.170(3)$ & $9.4073(11)$ & $19.4650(15)$ \\
\hline b $(\AA)$ & $8.7937(4)$ & $13.4118(4)$ & $9.3213(8)$ & $9.523(3)$ & $11.4997(13)$ & $14.1581(12)$ \\
\hline c $(\AA)$ & $17.2640(6)$ & $17.5796(5)$ & $15.6519(14)$ & 27.874(9) & $13.3594(16)$ & $8.6501(6)$ \\
\hline$\alpha\left(^{\circ}\right)$ & 90.00 & 90.00 & $100.882(3)$ & 90.00 & $90.033(4)$ & 90 \\
\hline$\beta\left(^{\circ}\right)$ & $90.303(3)$ & $106.8890(8)$ & $98.153(3)$ & $94.531(11)$ & $90.906(4)$ & $93.203(3)$ \\
\hline$\gamma\left({ }^{\circ}\right)$ & 90.00 & 90.00 & 106.181(3) & 90.00 & $102.274(4)$ & 90 \\
\hline $\mathrm{V}\left(\AA^{3}\right)$ & 1413.11(10) & 2741.87(13) & 1219.77(19) & $2955.9(15)$ & $1412.0(3)$ & 2380.1(3) \\
\hline $\mathrm{Z}$ & 2 & 4 & 2 & & 2 & 4 \\
\hline$D_{\text {calc }}\left(\mathrm{g} / \mathrm{cm}^{3}\right)$ & 1.462 & 1.453 & 1.520 & 1.489 & 1.602 & 1.815 \\
\hline$F(000)$ & 636 & 1224 & 564 & 1336 & 684 & 1268 \\
\hline$\mu\left(\mathrm{mm}^{-1}\right)$ & 1.155 & 1.113 & 1.351 & 1.1196 & 2.371 & 2.474 \\
\hline $2 \theta \leq\left(^{\circ}\right)$ & 60.50 & 54.16 & 55.02 & 55.02 & 55.06 & 49.98 \\
\hline $\begin{array}{l}\text { goodness-of-fit on } \\
F^{2}\end{array}$ & 1.033 & 1.027 & 110 & 10138 & 1.045 & 1.045 \\
\hline $\mathrm{R} 1, w \mathrm{R} 2[I>2 \sigma(I)]$ & 0.0329 & 0.0507 & 0.0283 & 0.0632 & 0.0722 & 0.0297 \\
\hline $\mathrm{R} 1, w \mathrm{R} 2$ (all data) & $=0.0618$ & 0.0230 & 0.0665 & 0.1568 & 1.212 & 0.0698 \\
\hline
\end{tabular}


- Transient metallaborane intermediates were stabilized by phenyl-chalcogenoborates $\mathrm{Li}\left[\mathrm{BH}_{3}(\mathrm{EPh})\right](\mathrm{E}=\mathrm{S}, \mathrm{Se}$ or Te $)$.

- Dimetallaboranes, analogues of pentaborane(11) and hexaborane(10), were synthesized and structurally characterized.

- Isolation and structural characterization of $\left[(\mathrm{Cp} * \mathrm{Ru})_{2} \mathrm{~B}_{4} \mathrm{H}_{6} \mathrm{Te}\right]$ provided a rare example of metallatelluraborane. 